\title{
Glucocorticoid-induced osteoporosis; management based on recent major international recommendations
}

\author{
Amila Rathnapala ${ }^{1}$, Noel Somasundaram ${ }^{2}$ \\ Sri Lanka Journal of Diabetes, Endocrinology and Metabolism 2013; 3: 80-83
}

\begin{abstract}
Osteoporosis is one of the important adverse effects of glucocorticoids causing significant disability due to fractures. Fracture is the presenting feature in $30-50 \%$ of patients with Glucocorticoid-Induced Osteoporosis (GIO). A rapid decline in bone mineral density (BMD) occurs in the first six months with the use of glucocorticoids.

We discuss the management strategies available to prevent and treat GIO based on the American College of Rheumatology (ACR) Guidelines (2010), American Society of Bone Mineral Research (ASBMR) recommendations (2011) and International Osteoporosis Foundation (IOF) Guidelines (2012).

All three guideline committees recommend counseling for lifestyle modification, risk assessment and adequate calcium and vitamin D supplementation.

The ACR expert panel has recommended the FRAX tool for the risk stratification. It considered the glucocorticoid dose as an average dose. However, there is strong evidence that the risk associated with glucocorticoid use is dose related. Considering that, IOF proposed a FRAX adjustment for the dose of glucocorticoids.

Even though there is evidence for the bisphosphonate therapy for patients with more than 50 years of age, there is a significant paucity of evidence for age less than 50 years group. ACR only recommends bisphosphonate therapy for the patients below 50 years with fragility fractures. But ASBMR recommends bisphosphonate therapy for patients below 50 years with $Z$-score less than -2 or if there is a significant risk of BMD loss. But the IOF has recommended the use of bisphosphonates based on clinical background and adjusted FRAX score.
\end{abstract}

\section{Introduction}

Osteoporosis is one of the important adverse effects of glucocorticoids causing significant disability due to fractures. We discuss the management strategies available to prevent and treat Glucocorticoid-Induced Osteoporosis (GIO) based on the American College of Rheumatology (ACR) Guidelines 2010 (1) and American Society of Bone Mineral Research (ASBMR) recommendations 2011(2) and International Osteoporosis Foundation (IOF) guidelines 2012 (3).

\section{Case vignette}

A 43 years old male with a past history of nephrotic syndrome secondary to minimal change disease in 2009, presents with proteinuria of $5 \mathrm{~g} /$ day and body swelling for one week. He had two similar episodes in the past which was treated with prednisolone $45 \mathrm{mg} /$ day for more than a year. He does not have a family history of fragility fractures.

He smokes 5 cigarettes daily for last 20 years (4 pack years).

His weight when he was not oedematous was $50 \mathrm{~kg}$ and the body mass index (BMI) was $19.5 \mathrm{~kg} / \mathrm{m}^{2}$.

Current episode was diagnosed as the third relapse of nephrotic syndrome and he was commenced on oral prednisolone $50 \mathrm{mg}$ daily with calcium lactate $300 \mathrm{mg}$ three times daily. He was also treated with captopril and losartan. His creatinine clearance was $96 \mathrm{ml} / \mathrm{min}$.

How should this patient be evaluated and managed to minimize the risk of fractures?

${ }^{1}$ Senior Registrar in Respiratory Medicine, National Hospital for Respiratory Diseases, Welisara, ${ }^{2}$ Consultant Endocrinologist, National Hospital of Sri Lanka, Colombo. 


\section{The clinical problem}

Glucocorticoid use is one of the leading risk factors for osteoporosis $(4,5)$ and fracture. It is known that fracture can be the presenting feature in $30-50 \%$ of patients with GIO (6). The loss of bone mineral density (BMD) in patients treated with glucocorticoids is biphasic. A rapid decline occurs in the first year and more slowly thereafter $(7,8)$. An increased risk of fractures has been reported with dosages of prednisolone (or equivalent) as low as 2.5-7.5 mg daily. This increased risk may relate more strongly to daily rather than cumulative doses of glucocorticoids $(9,10)$. In a retrospective study involving patients 18 to 64 years of age, continuous treatment with $10 \mathrm{mg}$ of prednisolone per day for more than 90 days, for various indications, as compared with no exposure to glucocorticoids demonstrated a 5-fold increased risk of hip and 5.9-fold increased risk of vertebral fracture. The combined effect of higher dose and longer duration, further increased relative risk to 7 -fold for hip and 17-fold for vertebral fractures (11). In addition, an increase in the risk of fractures has been reported with the use of inhaled glucocorticoids, as well as with alternate-day and intermittent oral regimens (5).

\section{Risk assessment}

Risk assessment is the most important step in the prevention and management of GIO.

The history should specifically focus to asses level of smoking, alcohol consumption, frequency of falls and family history of osteoporosis. The BMI calculation and neurological examination are essential components. It is always important to document the baseline height as asymptomatic vertebral fractures can be suspected with height monitoring.

It is also important to screen for secondary causes for osteoporosis such as renal impairment, chronic liver disease, hyperthyroidism and hypogonadism in clinically relevant cases.

The ACR expert panel has recommended the FRAX tool for the risk stratification (1). This tool uses updated, evidence-based estimates of absolute 10 year fracture risk (1). It was created for the purpose of integrating numerous risk factors into a clinically useful risk prediction model (12).

The FRAX tool considered the steroid dose as an average dose. However, there is good evidence that the risk associated with glucocorticoid use is dose related. It uses only the bone density value for the hip, but patients receiving glucocorticoids frequently lose bone mass in the spine before the hip, leading to a possible underestimation of fracture risk. Because of this some authors do not recommend FRAX as a tool for risk assessment in
GIO (13). But IOF expert committee has proposed a FRAX adjustment for the dose of steroids. The evidence came from the General Practice Research Database (GPRD) in UK, which has demonstrated that fracture risk is increased even with relatively low daily doses of glucocorticoids and rises further with increasing daily dose (14). The detailed FRAX adjustments are shown in Table 1.

\section{Table 1. The average FRAX adjustments for postmenopausal women and men aged $\geq 50$ years depending on the steroid dose}

The steroid dose

FRAX

adjustment

$<2.5 \mathrm{mg} /$ day prednisolone or its

0.65

equivalent for hip fracture

$\geq 7.5 \mathrm{mg} /$ day prednisolone or its

equivalent for hip fracture

$<2.5 \mathrm{mg} /$ day prednisolone or

its equivalent for major osteoporotic fracture

$\geq 7.5 \mathrm{mg} /$ day prednisolone or its equivalent for major osteoporotic fracture

\section{Strategies for prevention of GIO}

Smoking cessation, avoidance of alcohol intake, regular physical exercises, calcium and vitamin $\mathrm{D}$ supplementation are basic strategies to prevent GIO, although the evidence for their effects on fracture risk is weak. The recommended calcium intake (supplement plus oral intake) is $1200-1500 \mathrm{mg} /$ day and the recommended dose of vitamin D is 800-1000 units per day. A higher dose is recommended as glucocorticoids interfere with vitamin D absorption (15).

In addition to the above, all the guidelines recommend the use of smallest dose of glucocorticoid for the shortest duration possible as there may be no dose of steroids that does not accelerate bone loss or increase fracture risk (16). Alternative strategies should be considered to minimize the steroid dose. Different routes (eg. topical, inhalational) of administration or different formulations may be considered in some occasions. Use of alternative immunosuppressive treatment (eg. azathioprin) may enable reduction of the dose of glucocorticoids.

\section{Monitoring patients receiving steroids}

Assessment of compliance to osteoporosis medication, assessment of incidence of fragility fractures and annual height measurement are essential components of monitoring. In some, serial BMD testing can be done. 


\section{Pharmacotherapy for the management and prevention of GIO}

The IOF committee has recommended their preventive strategies based on clinical and adjusted FRAX score. Even though there is evidence for the recommendations for patients above 50 years of age, there is a significant paucity of evidence for the age group below 50 years (3).

IOF has proposed several principles of GIO management (3). Firstly the bone protection therapy should be started at the onset of steroid therapy and should be withdrawn when the steroid therapy is terminated. Calcium and vitamin D supplementation with adequate dietary intake should be maintained. Alendronate, etidronate, risedronate, zoledronic acid and teriparatide can be used as the front-line therapeutic options for the majority of patients.

In patients who are 50 years and younger, the recommendations are varied across the three guidelines, since data on pharmacological interventions are sparse. But evidence suggests that the fracture risks among young are less compared to the older people. ACR only recommends therapy for the patients who have fragility fractures, however the American Society for the Bone Mineral Research (ASBMR) recommends GIO therapy for patients less than 50 years of age with $\mathrm{Z}$ score less than 2 or if there is a significant risk of BMD loss (2). IOF recommends bone protective therapy for young people with previous fragility fractures or who are on high dose of glucocorticoids (3).

\section{Conclusion and recommendations}

This patient has several risk factors for GIO, which include current smoking, past history of steroid use and current high dose prednisolone treatment. It is essential to advise and support him quit smoking and to counsel him to have adequate calcium and vitamin D intake with normal protein diet.

At the time of admission, he should undergo DXA (Dual Energy X ray Absorptiometry) scan to assess the BMD.

He should be treated with oral calcium salts 1200 $1500 \mathrm{mg}$ daily and oral vitamin D 800-1000 IU per day. There are very few data on the use of glucocorticoids in men at this age group. But considering the disease which requires high doses of steroids for longer duration, he should be treated with bisphosphonates, according to the IOF recommendations.

Alendronate is the first line therapy in Sri Lankan set up, since it is freely available in the public health sector. The dose should be either $70 \mathrm{mg}$ weekly or $10 \mathrm{mg}$ daily. Half dose of alendronate or any other bisphosphonate is not recommended to prevent bone loss in GIO.
The duration of the therapy should be decided carefully because most of the studies done with bisphosphonates have been done for short periods and new potential adverse effects of long-term therapy such as osteonecrosis of the jaw and atypical subtrochanteric fractures have been recognized $(17,18)$. As studies for the prevention and treatment of GIO lasted no more than 3 years, it is the opinion of the ASBMR Professional Practice Committee that patients requiring longer-term steroid therapy be evaluated for substitution of bisphosphonate treatment with teriparatide, or be considered for treatment with teriparatide initially, followed by treatment with a bisphosphonate (2). But IOF has shown that the benefit of continuation of bisphosphonates is far more than the risk (3). Even though some experts recommend drug holidays when using for longer durations, the current data concerning the appropriateness of it is inadequate due to the small number of studies.

\section{Limitations of the recommended GIO therapy}

ACR and ASBMR expert panels are not clear about the individual doses of bisphosphonates. GIO therapies for patients with chronic renal insufficiency are also not adequately discussed. The systemic review of most of the guidelines were only restricted to the European countries and America.

\section{Acknowledgment}

We would like to thank Professor Sarath Lekamwasam for his kind advice and assistance.

\section{References}

1. Grossman JM, Gordon R, Ranganath VK, Deal C, Caplan L, Chen W, et al. American College of Rheumatology 2010 recommendations for the prevention and treatment of glucocorticoid-induced osteoporosis. Arthritis Care and Research 2010; 62(11): 1515-26.

2. Hansen KE, Wilson HA, Zapalowski C, Fink HA, Minisola $\mathrm{S}$, Adler RA. Uncertainties in the prevention and treatment of glucocorticoid-induced osteoporosis. Journal of the American Society for Bone and Mineral Research 2011; 26(9): 1989-96.

3. Lekamwasam S, Adachi JD, Agnusdei D, Bilezikian J, Boonen S, Borgstrom F, et al. A framework for the development of guidelines for the management of glucocorticoidinduced osteoporosis. Osteoporosis International 23 (9): 2257-76.

4. Canalis E, Mazziotti G, Giustina A, Bilezikian JP. Glucocorticoid-induced osteoporosis: pathophysiology and therapy. Osteoporosis International 2007; 18(10): 1319-28.

5. Weinstein RS. Glucocorticoid-induced osteoporosis. In: Rosen C, editor. The ASBMR primer on the metabolic bone diseases and disorders of mineral metabolism. 7 ed. Washington, DC: ASBMR; 2008. p. 267-72. 
6. Adler RA, Curtis JR, Saag K, Weinstein RS. Glucocorticoidinduced osteoporosis. In: Marcus R, Feldman D, Nelsen DA, Rosen CJ, editors. Osteoporosis. 3 ed. San Diego, CA: Elsevier-Academic Press; 2008. p. 1135-66.

7. Lane NE, Lukert B. The science and therapy of glucocorticoid-induced bone loss. Endocrinology and Metabolism Clinics of North America 1998; 27(2): 465-83.

8. LoCascio V, Bonucci E, Imbimbo B, Ballanti P, Adami S, Milani S, et al. Bone loss in response to long-term glucocorticoid therapy. Bone Miner 1990; 8(1): 39-51.

9. van Staa TP, Leufkens HG, Abenhaim L, Zhang B, Cooper C. Oral corticosteroids and fracture risk: relationship to daily and cumulative doses. Rheumatology (Oxford). 2000; 39(12):1383-9.

10. Sambrook PN, Eisman JA, Yeates MG, Pocock NA, Eberl $\mathrm{S}$, Champion GD. Osteoporosis in rheumatoid arthritis: safety of low dose corticosteroids. Annals of the Rheumatic Diseases 1986; 45(11): 950-3.

11. Steinbuch M, Youket TE, Cohen S. Oral glucocorticoid use is associated with an increased risk of fracture. Osteoporosis International 2004; 15(4): 323-8.

12. Dawson-Hughes B, Tosteson AN, Melton LJ, 3rd, Baim S, Favus MJ, Khosla S, et al. Implications of absolute fracture risk assessment for osteoporosis practice guidelines in the USA. Osteoporosis International 2008; 19(4): 449-58.
13. Weinstein RS. Clinical practice. Glucocorticoid-induced bone disease. The New England Journal of Medicine 2011; 365(1): 62-70.

14. van Staa TP, Leufkens HG, Cooper C. The epidemiology of corticosteroid-induced osteoporosis: a meta-analysis. Osteoporosis International 2002; 13(10): 777-87.

15. Holick MF. Optimal vitamin D status for the prevention and treatment of osteoporosis. Drugs and Aging 2007; 24(12):1017-29.

16. van Staa TP, Geusens P, Pols HA, de Laet C, Leufkens HG, Cooper C. A simple score for estimating the long-term risk of fracture in patients using oral glucocorticoids. QJM: monthly. Journal of the Association of Physicians 2005; 98(3): 191-8.

17. MacLean C, Newberry S, Maglione M, McMahon M, Ranganath V, Suttorp M, et al. Systematic review: comparative effectiveness of treatments to prevent fractures in men and women with low bone density or osteoporosis. Annals of Internal Medicine 2008; 148(3): 197-213.

18. Lefebvre C, Eisinga A, McDonald S, Paul N. Enhancing access to reports of randomized trials published worldwide - the contribution of EMBASE records to the Cochrane Central Register of Controlled Trials (CENTRAL) in The Cochrane Library. Emerging Themes in Epidemiology 2008; 5: 13. 